\title{
Component-oriented acausal modeling of the dynamical systems in Python language on the example of the model of the sucker rod string
}

\author{
Volodymyr B Kopei ${ }^{\text {Corresp., }}{ }^{1}$, Oleh R Onysko ${ }^{1}$, Vitalii G Panchuk ${ }^{1}$ \\ ${ }^{1}$ Department of Computerized Mechanical Engineering, Ivano-Frankivsk National Technical University of Oil and Gas, Ivano-Frankivsk, Ukraine \\ Corresponding Author: Volodymyr B Kopei \\ Email address: volodymyr.kopey@nung.edu.ua
}

Typically, component-oriented acausal hybrid modeling of complex dynamic systems is implemented by specialized modeling languages. A well-known example is the Modelica language. The specialized nature, complexity of implementation and learning of such languages somewhat limits their development and wide use by developers who know only general-purpose languages. The paper suggests the principle of developing simple to understand and modify Modelica-like system based on the general-purpose programming language Python. The principle consists in: (1) Python classes are used to describe components and their systems, (2) declarative symbolic tools SymPy are used to describe components behavior by difference or differential equations, (3) the solution procedure uses a function initially created using the SymPy lambdify function and computes unknown values in the current step using the know n values from the previous step, (4) Python imperative constructs are used for simple events handling, (5) external solvers of differential-algebraic equations can optionally be applied via the Assimulo interface, (6) SymPy package allows to arbitrarily manipulate model equations, generate code and solve some equations symbolically. The basic set of mechanical components (1D translational "mass", "spring-damper" and "force") is developed. The models of a sucker rods string are developed and simulated using these components. The comparison of results of the sucker rod string simulations with practical dynamometer cards and Modelica results verify the adequacy of the models. The proposed approach simplifies the understanding of the system, its modification and improvement, adaptation for other purposes, makes it available to a much larger community, simplifies integration into third-party software. 
2 Component-oriented acausal modeling of the

3 dynamical systems in Python language on the

4 example of the model of the sucker rod string

5

6

7

8

9

10

11

12

13

14

15

16

17

18

19

20

21

22

23

24

25

26

27

28

29

30

31

32

33

34

35

36

37

38

Volodymyr Bohdanovych Kopei ${ }^{1}$, Oleh Romanovych Onysko ${ }^{1}$, Vitalii Georgievich Panchuk ${ }^{1}$

${ }^{1}$ Department of Computerized Mechanical Engineering, Ivano-Frankivsk National Technical University of Oil and Gas, Ivano-Frankivsk, Ukraine

Corresponding Author:

Volodymyr Kopei ${ }^{1}$

15 Karpatska Street, Ivano-Frankivsk, 76019, Ukraine

Email address: volodymyr.kopey@nung.edu.ua

\section{Abstract}

Typically, component-oriented acausal hybrid modeling of complex dynamic systems is implemented by specialized modeling languages. A well-known example is the Modelica language. The specialized nature, complexity of implementation and learning of such languages somewhat limits their development and wide use by developers who know only general-purpose languages. The paper suggests the principle of developing simple to understand and modify Modelica-like system based on the general-purpose programming language Python. The principle consists in: (1) Python classes are used to describe components and their systems, (2) declarative symbolic tools SymPy are used to describe components behavior by difference or differential equations, (3) the solution procedure uses a function initially created using the SymPy lambdify function and computes unknown values in the current step using the known values from the previous step, (4) Python imperative constructs are used for simple events handling, (5) external solvers of differential-algebraic equations can optionally be applied via the Assimulo interface, (6) SymPy package allows to arbitrarily manipulate model equations, generate code and solve some equations symbolically. The basic set of mechanical components (1D translational "mass", "spring-damper" and "force") is developed. The models of a sucker rods string are developed and simulated using these components. The comparison of results of the sucker rod string simulations with practical dynamometer cards and Modelica results verify the adequacy of the models. The proposed approach simplifies the understanding of the system, its modification and improvement, adaptation for other purposes, makes it available to a much larger community, simplifies integration into third-party software. 
39

40

41

42

43

44

45

46

47

48

49

50

51

52

53

54

55

56

57

58

59

60

61

62

63

64

65

66

67

68

69

70

71

72

73

74

75

76

77

78

\section{Introduction}

As is known, component-oriented simulation modeling is based on the separation of a complex system model into simple components. The component describes the mathematical model of the corresponding physical object (mass, spring, electrical resistance, hydraulic resistance, hydraulic motor, etc.), which is formulated as an algebraic, differential or difference equation. The components are connected through ports (pins, flanges), which define a set of variables for the component interaction (Elmqvist, 1978; Fritzson, 2015). The components and ports are stored in software libraries. Usually, it is possible to develop new components. The multi-domain modeling allows using together of components that differ in physical nature (mechanical, hydraulic, electric, etc.). The component-oriented modeling can be based on causal modeling or acausal modeling (Fritzson, 2015). In the first case, the component receives the $x$ signal at the input, performs a certain mathematical operation $f(x)$ on it and returns the $y$ result to the output. In this case, the modeling is realized by imperative programming by assigning the value of the $f(x)$ expression to the $y$ variable. In the second case, the signal of the connected components can be transmitted in two directions. Such modeling is realized by declarative programming by solving the equation $y=f(x)$, where the unknown can be $x$ or $y$. Here, the variables $x$ and $y$ are some physical quantities, and the equation $y=f(x)$ is the physical law that describes their relationship. It allows us to simplify the creation of the model, to focus on the physical formulation of the problem, but not on the algorithm for solving it. It is also possible to avoid errors that are typical for imperative programming.

The behavior of these models is most often described by a system of ordinary differential equations (ODEs) or a differential-algebraic system of equations (DAEs), which are solved by the finite difference method - a numerical method based on the replacement of differential operators by difference schemes. As a result, the system of differential equations is replaced by the system of algebraic equations.

The solution of non-stationary problems by the finite difference method is the iterative process - there is a solution of the stationary problem for the given time point at each iteration. Explicit and implicit difference schemes are used for this purpose. Explicit schemes immediately find unknown values, using information from the previous iterations. Using the implicit scheme requires the solution of a difference equation because unknown values can be in the right and left sides of the equation. The explicit Euler difference scheme is simple to implement, but it often has numerical instability and low accuracy. The analysis of the Euler method is described in detail in (Atkinson, 1989). To improve accuracy and stability it is desirable to apply modified Euler methods, such as the Runge-Kutta method (Runge, 1895).

For simulations of complex dynamic multi-domain systems such specialized equation-based modeling languages are developed: Dymola (Elmqvist, 1978), APMonitor (Hedengren et al., 2014), ASCEND (Piela, McKelvey \& Westerberg, 1993), gPROMS (Barton \& Pantelides, 1994), Modelica (Fritzson \& Engelson, 1998), MKL, Modelyze (Broman, 2010). Among them, Modelica is the most popular free language for component-oriented modeling of such systems.

Its main features: free, object-oriented, declarative, focused on hybrid (continuous and discrete) 
79

80

81

82

83

84

85

86

87

88

89

90

91

92

93

94

95

96

97

98

99

100

101

102

103

104

105

106

107

108

109

110

111

112

113

114

115

116

117

118

component-oriented modeling of complex multi-domain physical systems, it supports the construction of hierarchical models, is adapted for visual programming and widely used for research in various fields (Fritzson, 2015). Free Modelica Standard Library has about 1280 components. There are free and commercial simulation environments for Modelica OpenModelica, JModelica.org, Wolfram SystemModeler, SimulationX, MapleSim, Dymola, LMS Imagine.Lab AMESim.

The known problem of specialized languages is the complexity of the modifications and improvements and a relatively small community of developers. They are not very well suited for experimenting with evolutions of modeling capabilities (Elmqvist, Henningsson \& Otter, 2016). In particular, developers of some languages have encountered the problem of variable structure systems modeling where the structure and number of equations can change at run-time (Fritzson, Broman \& Cellier, 2008; Nikolić, 2016). Some problems can be solved by using interfaces to general-purpose languages (Akesson et al., 2010; Hedengren et al., 2014). But it is usually more difficult to learn a new language than to learn a component or a library of a familiar programming language.

As a rule, general-purpose languages, in comparison with specialized languages, are more widespread, easy to learn thanks to typical imperative and object-oriented constructs, have wider applicability, better interoperability with the third party software and a large number of heterogeneous packages. Therefore, the mentioned problems are less common in modeling systems that are based on general-purpose programming languages: PyDSTool (Clewley et al., 2007) - Python-based Dynamical Systems Toolkit with support for symbolic manipulation, hierarchical structures and hybrid models; Ariadne - a C++ library for formal verification of cyber-physical systems, using reachability analysis for nonlinear hybrid automata (Benvenuti et al., 2014); Assimulo - Python-package that combines a variety of different ODE/DAE solvers via a common high-level interface (Andersson, Führer \& Åkesson, 2015); DAE Tools equation-based object-oriented modeling, simulation and optimization software with hybrid approach (Nikolić, 2016); Modia.j1 — Modelica-like language that is directly defined and implemented with Julia's meta-programming constructs and is designed tightly together with the symbolic and numeric algorithms (Elmqvist, Henningsson \& Otter, 2016); SimuPy - a Python framework for simulating interconnected dynamical system models (Margolis, 2017); Sims.j1 a Julia package for equation-based hybrid modeling and simulations, similar to Modelyze (Short, 2017); GEKKO — a Python package for machine learning and optimization of mixed-integer and differential algebraic equations (Beal et al., 2018). However, most of these systems either have a complex code that is difficult to understand and modify (e.g. have their own symbolic processors), or use state-of-the-art ODE/DAE solvers that are implemented in low-level languages, which rarely allow modification to an untrained user. The implementation, modification and improvement of such systems can be simplified if the difference equations are used to describe the model instead of differential equations. In addition, difference equations are also often used to model dynamical systems. In general, the modeling system should allow various types of equations. 
119 The advantages of modeling systems based on general-purpose programming languages are 120 described in detail in papers (Nikolić, 2016; Elmqvist, Henningsson \& Otter, 2016). Python 121 language (Van Rossum \& Drake, 1995) is a good choice mainly due to its features: multi122 paradigm, object-oriented, intuitive with code readability and improved programmer's

123 productivity, highly extensible, portable, open-source, large community and extensive libraries 124 as mathematical libraries SymPy and SciPy. SymPy is a Python library for symbolic

125 mathematics (Meurer et al., 2017). SciPy is a fundamental library for scientific computing (Jones 126 et al., 2001).

127 This work suggests the principle of developing simple to understand and modify Modelica-like 128 system based on the general-purpose programming language Python. The principle consists in: 129 (1) Python classes are used to describe components and their systems, (2) declarative symbolic 130 tools SymPy are used to describe components behavior by difference or differential equations, 131 (3) the solution procedure uses a function initially created using SymPy lambdify and computes unknown values in the current step using the known values from the previous step, (4) Python imperative constructs are used for simple events handling, (5) external DAEs solvers can optionally be applied via the Assimulo interface, (6) SymPy package allows to arbitrarily manipulate model equations, generate code and solve some equations symbolically. The principle of the system is described by examples of models of a sucker rod string that is used in the oil industry to connect surface and downhole components of a rod pumping system.

138

Methods and implementation

\section{Description of modeling principles in Modelica language}

First, we describe the modeling principles in Modelica using an example of a simple mechanical translational oscillator. The oscillator consists of such components as Mass, SpringDamper and Fixed (Fig. 1). The SpringDamper component is designed to simulate the elasticdamper properties of the damped oscillator. The Mass component simulates the inertial properties of the oscillator. The Fixed component simulates the fixed point of the oscillator. The module code that describes this model is explained below (Listing S1). In order to simplify the model, these classes differ slightly from the corresponding classes of the standard Modelica library (Fritzson, 2015). A class in Modelica describes the set of similar objects (components). The Flange class describes the concept of a mechanical flange. Its real-type $\mathrm{s}$ variable corresponds to the absolute position of the flange. Its value should be equal to the value of the s variables of other flanges connected to this flange. The real-type $f$ variable corresponds to the force on the flange. It is marked by the flow keyword, which means that the sum of all forces at the connection point is zero.

connector Flange // class-connector

Real s; // variable (positions at the flange are equal)

flow Real f; // variable (sum of forces at the flange is zero) 
159

160

161

162

163

164

165

166

167

168

169

170

171

172

173

174

175

176

177

178

179

180

181

182

183

184

185

186

187

188

189

190

191

192

193

194

195

196

197

198

199

200

end Flange;

The Fixed class describes the concept of a fixed component with one flange, for example, fixed1 (Fig. 1). It has the real-type s0 variable, which corresponds to the absolute position of the flange, and the $f$ lange object of the $F$ lange class, designed to connect this component to others. The s 0 variable is marked by the parameter keyword, which means that it can be changed only at the start of the simulation. After the equation keyword, an equation describing the behavior of this component is declared - the flange object position must be equal to the $\mathrm{s} 0$ value.

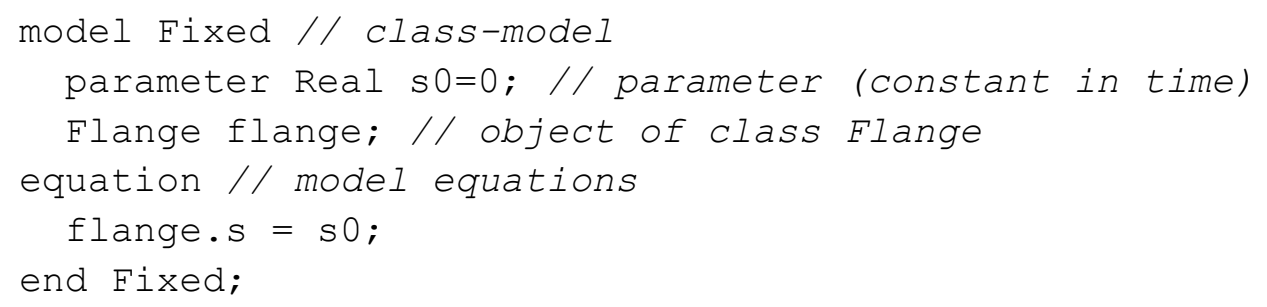

The Transl class describes an abstract component that has two flanges _ flange_a and flange_b. It is the base class for mechanical translational components with two flanges.

partial model Transl // class-model

Flange flange_a; // object of class Flange

Flange flange_b; // object of class Flange

end Transl;

The Mass class inherits the Transl class and describes the sliding mass with inertia. The example of such component is mass 1 (Fig. 1). The extends Transl command means inheriting members of the Transl class in such a way that they become members of the Mass class. That is, the Mass component will also have two flanges (flange_a and flange_b). In addition, this class has the $m$ parameter (mass) and the variables: $s$ (position), $v$ (speed), a (acceleration). The expression start $=0$ is the default initial condition. After the equation keyword the system of the differential and algebraic equations, which describes the behavior of this component, is given. The der keyword means the derivative with respect to time $t(v=\mathrm{d} s / \mathrm{d} t$, $a=\mathrm{d} v / \mathrm{d} t)$.

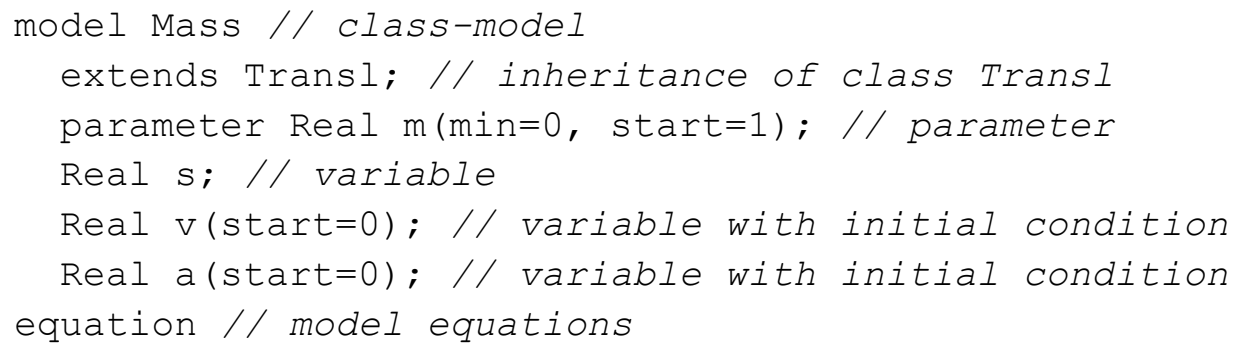


201

202

203

204

205

206

207

208

209

210

211

212

213

214

215

216

217

218

219

220

221

222

223

224

225

226

227

228

229

230

231

232

233

234

235

236

237

238

239

240

241

242

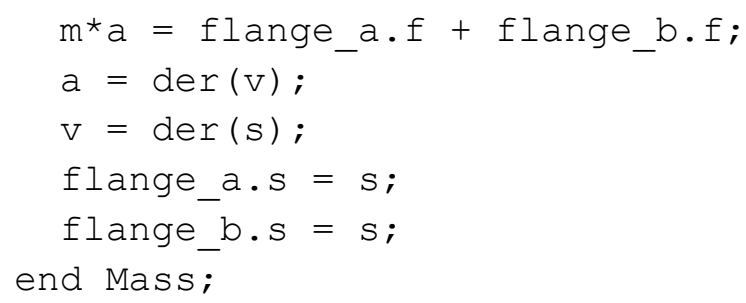

The SpringDamper class inherits the Transl class and describes the linear 1D translational spring and damper in parallel (Listing S1). The example of such component is springDamper1 (Fig. 1). The class has the parameters c (spring constant), d (damping constant) and the variables $s_{-} r e l$ (relative position), $v_{-} r e l$ (relative speed), $f$ (force at flange_b). After the equation keyword the system of differential-algebraic equations of this component is given.

The Oscillator class describes the spring-mass system (Fig. 1). It contains three components mass1, spring1, fixed1, which are described by the classes Mass, SpringDamper and Fixed, respectively. The values of parameters and initial conditions of these components are shown in round brackets.

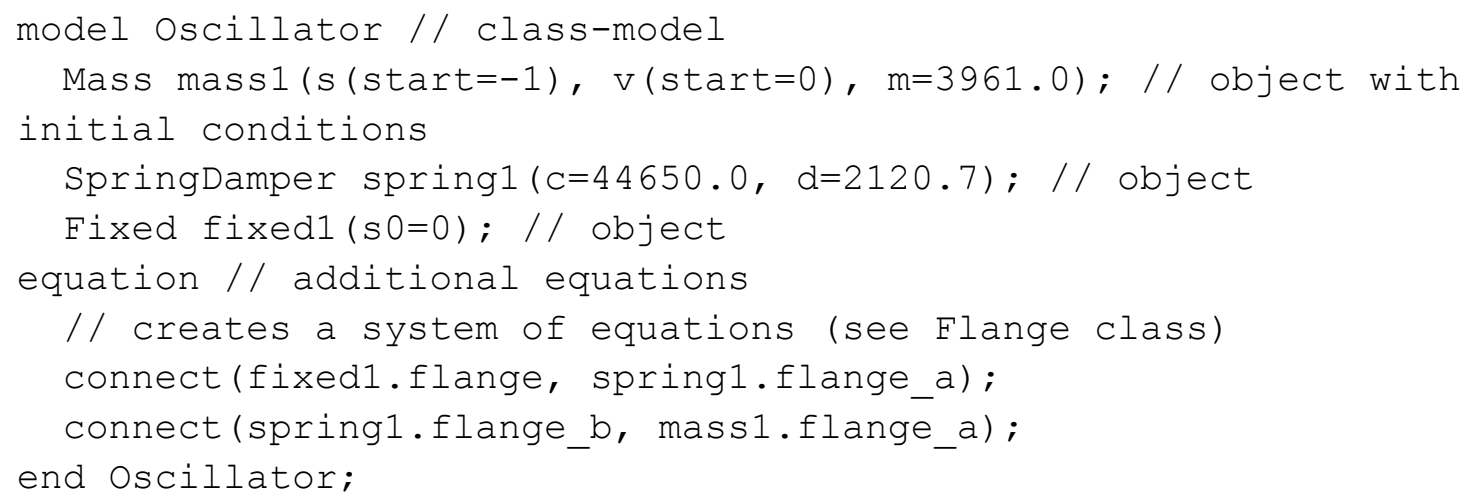

The additional equations, which are obtained from component connections, are given after the equation keyword. For example the connect ( fixedl. flange, spring1.flange_a) command connects the flanges of the fixed1 and spring1 components and creates the additional system of equations:

fixedl.flange.s = springl.flange_a.s;

fixed1.flange.f $=$-springl.flange_a.f

The model code can be prepared using any text editor or the Modelica Development Tooling (MDT) module (Pop et al., 2006) of the Eclipse development environment. Simulation of a model requires the OpenModelica environment (Fritzson et al., 2005). To start calculations and to plot the curve, which describes the position of mass 1 component with time, enter the following into the OpenModelica Shell: 
243

244

245

246

247

248

249

250

251

252

253

254

255

256

257

258

259

260

261

262

263

264

265

266

267

268

269

270

271

272

273

274

275

276

277

278

279

280

281

282

loadModel (Modelica)

loadFile ("Pycodyn.mo")

simulate (Pycodyn.Oscillator, stopTime=10)

plot (mass1.s)

The typical stages of translating and executing a Modelica model are (Fritzson, 2015): translation (obtaining a flat set of equations, constants, variables and function definitions from Modelicacode), analysis (equations sorting, convert the coefficient matrix into block lower triangular form), optimization (elimination of most equations, converting equations to assignment statements), code generation (obtaining a C-code), compilation (obtaining an executable) and simulation. However, there are alternative ways of executing Modelica (Fritzson, 2015).

\section{Description of modeling principle in Python}

The principle of component-oriented modeling in Python is described below and an example of the implementation of a similar oscillator model is shown.

1. Components are described by Python classes that are structurally similar to Modelica classes and have the following attributes: constant parameters and SymPy symbols (analogs of parameters and variables in Modelica), SymPy symbolic equations (difference or DAE), pins for connecting components into a system (analogs of connectors in Modelica). A dynamic system consisting of components is also described by a Python class which attributes are a list of components and equations (together with additional equations for connecting components).

2. If the dynamic behavior of the components is described by difference equations, then the user must describe these equations in the class by replacing the derivatives with the selected difference scheme (e.g. by the Euler method).

3. The initial conditions are substituted into these difference equations and unknowns are found by solving a system of nonlinear equations at each step or using a function that was initially created using the SymPy lambdify function (translates a SymPy expression into an equivalent numeric function) and calculate unknown values at current step from the known values from the previous step without the need to solve the equations.

4. At each step, the if statement checks for discrete events that depend on state or time. During event handling, initial conditions, components, or equations can be changed.

5. If the dynamic behavior of the components is described by DAEs, then the Assimulo interface to the DAEs solvers is used, which has an effective discontinuity handling procedure.

6. The SymPy package allows arbitrary manipulation of model equations and code generation.

You can solve some algebraic or differential equations symbolically. The DAE system of equations should be simplified, transformed into an ODE, and solved by the SymPy dsolve function.

Now we will develop the pycodyn module with similar components in Python (Listing S2).

The behavior of the components will be described using the difference equations. For simplicity,

Peer) Comput. Sci. reviewing PDF | (CS-2019:03:35946:2:0:NEW 14 Sep 2019) 
283 we will use the Euler method. As a result, the system of components connected by flanges will

284 be described by the system of the difference equations.

285 First, import the sympy module and the standard mathematical module math. It is important to 286 distinguish the functions of these modules.

287

288

289

from sympy import *

import math

290

291

Create the global variable dt (time step).

292

293

$d t=0.1$

294

295

If you only need to obtain the system of equations in a symbolic form, then this variable must be

296

297

298

299

300 an instance of the Symbol class from the sympy module:

$d t=S y m b o l(' d t ')$

301

Translational1D is the basic class of mechanical 1D components with translational motion.

302

The init method is called when an object of this class is created and has two parameters - the name of the component (name) and the dictionary of its attributes (args). For

303 component attribute naming, we use the following notation. At the beginning of the name, the $x$,

304 $\mathrm{v}, \mathrm{a}, \mathrm{f}$ symbols mean position, speed, acceleration and force, respectively. At the end of the

305 name, the $p$ symbol means the value at time $t-d t$. The numerical index at the end corresponds

306 to the flange number. To distinguish the variables of various components in the system, each of

307 them begins with the name of the component followed by the symbol "_.". For example, the

308 $\mathrm{s} 1 \mathrm{x} 2 \mathrm{p}$ name means the position of the second flange of the s1 component at time $t-d t$. The init method for each name-value pair of the dictionary args (except name and self)

310 creates SymPy variables. The symbolic variable of the Symbol class is created if its value is not known. In another case, the numeric variable of the Number class is created. The self.eqs list contains the component equations, and the self.pins list contains the component flanges. Each equation is created using SymPy class Eq. Each flange is described by

314 a dictionary, which keys are $\mathrm{x}, \mathrm{xp}, \mathrm{f}$, and the values are the corresponding attributes of the component (see Mass, SpringDamper, Force classes). The pinEqs method returns a list of equations for the component flange that is connected to the flanges of the other components. It

317 has the pindex parameter - the index of the flange (e.g. 0), and the pins parameter - the

318 list of other components flanges. Always the positions of the mechanical 1D translational 319 component on the flange are equal, and the sum of the forces on this flange is zero. For example, 320 if the flange 2 of an $\mathrm{s} 1$ component is connected to the flange 1 of an $\mathrm{m} 1$ component then

321 pinEqs method of the $\mathrm{s} 1$ component returns the list of equations [ $\mathrm{s}{ }_{-} \mathrm{x} 2==\mathrm{m} 1 \mathrm{x} 1$,

322 s1_x $\left.2 p==m 1 \_x 1 p, s 1 \_f 2==-m 1 \_f 1\right]$. 
323

324

325

326

327

328

329

330

331

332

333

334

335

336

337

338

339

340

341

342

343

344

345

346

347

348

349

350

351

352

353

354

355

356

357

358

359

360

361

362

363

364

365

366

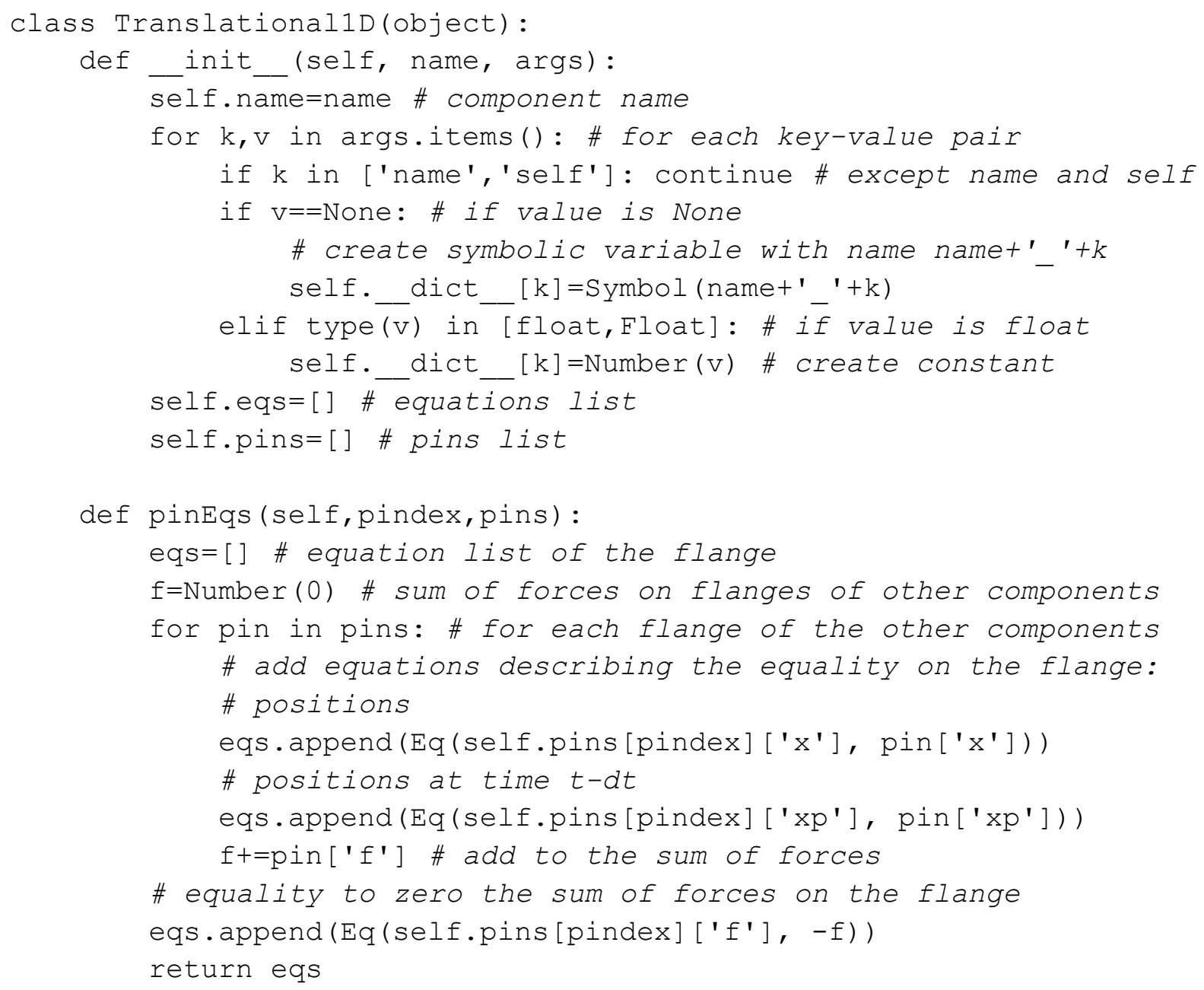

The Mass class describes the mass concentrated at a point, which has a translational motion. It inherits Translational1D class. The _ init_ constructor calls the constructor of the base class Translational1D and sends to it the parameters name and locals (). The latter is a dictionary of local variables self, name, $m, x, x p, v, v p, a, f 1, f 2$.

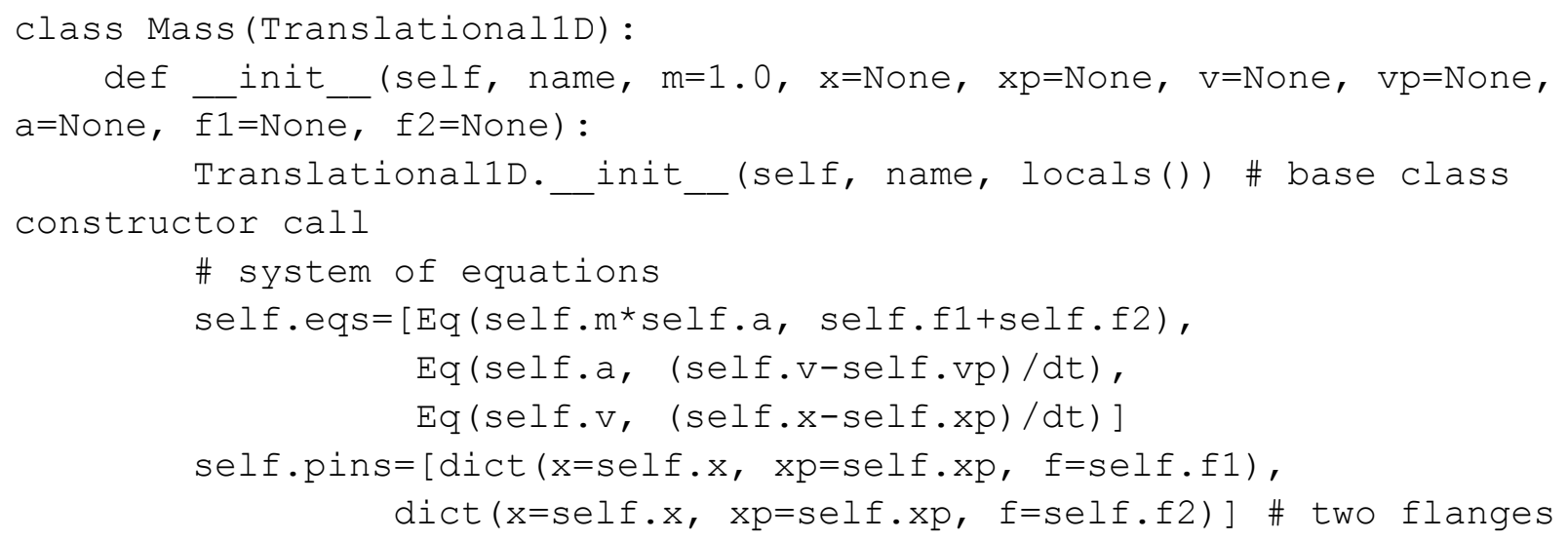


367

368

369

370

371

372

373

374

375

376

377

378

379

380

381

382

383

384

385

386

387

388

389

390

391

392

393

394

395

396

397

398

399

400

401

402

403

404

405

The behavior of this component is described by a system of equations self . eqs. For example, for an $\mathrm{m} 1$ component:

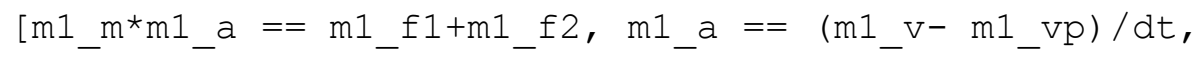
$\left.m 1 \_v==\left(m 1 \_x-m 1 \_x p\right) / d t\right]$

A list of additional equations can be generated for each component flange using the pinEqs method described above. The first element of the self.pins list is the dictionary

dict $(x=\operatorname{sel} f . x, x p=s e l f . x p, f=s e l f . f 1)$, which means that the $x, x p$ positions on the flange will be equal to the self $. x, \operatorname{sel} f . x p$ attributes of this component respectively, and the force $f$ on the flange will be equal to the self. $f 1$ attribute. The same applies to the second element of the list.

The SpringDamper class (Listing S2) describes the translational 1D spring and damper, which are connected in parallel. It inherits Translational1D class. In addition to the attributes described above, it has the following attributes: spring constant $\mathrm{c}$, damping constant $\mathrm{d}$, relative velocity between flanges vrel. The behavior of this component is described by a system of equations self.eqs. E.g. for an s1 component:

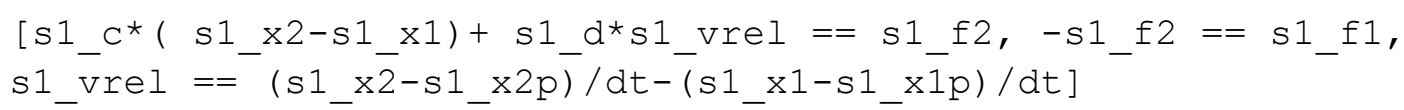

This component also has two flanges and it is possible to generate a list of additional equations using the pinEqs method.

The Force class (Listing S2) describes a 1D force with a translational motion of the application point. The value of the $f$ force can be constant or variable. It inherits the Translational1D class and has one flange.

The System class (Listing S2) describes the system of components connected by flanges. The constructor _ init__ gets two parameters — the list of components els and the list of additional equations eqs, which usually are created using pinEqs method. The system components are stored in the self.els list and the self.elsd dictionary. The list self .eqs contains all system equations and is created by joining the equations of all components with additional equations eqs.

The solve method of this class solves a stationary problem. It returns the solution of a system of equations with conditions i cs - a dictionary with known values of variables. To solve a system of equations, it can use the SymPy solve function, but its algorithm is very slow. It is possible to use fast algorithms for solving equations, e.g. the function

scipy.optimize. root from the SciPy library, which supports many effective methods for solving nonlinear systems of equations. In this case, the call of the SymPy function

Peer] Comput. Sci. reviewing PDF | (CS-2019:03:35946:2:0:NEW 14 Sep 2019) 
406 solve (eqs) must be replaced with the call of the self. solveN (eqs) method, which

407 adapts the system of equations for SciPy and solves it using scipy . optimize.root.

408 The solveDyn method solves a non-stationary problem. It receives three parameters - the

409 dictionary with initial state state, the final time value timeEnd and the $\mathrm{nBC}$ function that

410 returns the dictionary to update the state. First, the time variable $t$ is assigned an initial value. In

411 the while loop with the condition $t<t i m e E n d$, the following instructions are executed:

412 previous step variables ( $\mathrm{xp}, \mathrm{x} 1 \mathrm{p}, \mathrm{vp}$, etc.) are assigned the values of the initial state state,

413 the values of the boundary conditions are updated, the system of equations is solved by calling

414 the self.solve method, solutions are assigned to the dictionary state, the results are saved,

415 the time value increases by $d t$. After the loop is completed, the method returns the results as $\mathrm{T}$

416 and Res lists. These results can be represented in the form of plots using the matplotlib

417 library.

418 But the use of the self.solve method can be acceptable only for very frequent discontinuities

419 that require the re-creation of equations. Since at each step this method creates and solves a

420 system of nonlinear equations, the calculations can be very time-consuming. In most cases, it

421 should be replaced by the solvN method, which at each step finds unknown values by passing

422 the values found in the previous step to the ceqs $f$ method. At the beginning of the simulation

423 and after the discontinuities, this method must be created using SymPy lambdify function

424 that transforms SymPy expressions to lambda functions which can be used to calculate

425 numerical values very fast. This is done in the createCurEqs method.

426 Event processing is performed at the end of each step by calling the user-defined event handling

427 method event (state). In it, the if statement checks a determined condition with state.

428 If the result is True, then the event is handled, for example, new boundary conditions are

429 created and createCurEqs is called. You can easily implement modeling of variable structure

430 systems by calling in the event method the constructor of the System class (with new values

431 of els, eqs) and the createCurEqs method.

432 If differential equations are used in the components, then the functions and their derivatives

433 should be distinguished. The presence of the symbol " $D$ " in the name of the variable means

434 derivative. For example, $m 1_{-} \mathrm{Dx}$ is a derivative of $\mathrm{m}{ }_{1} \mathrm{x}$. Class descriptions of such components

435 will be more like Modelica classes (Table 1). In this case, to solve the DAEs in the form $0=F(t$,

$436 y, y^{\prime}$ ), the solveDAE method from the pycodynDAE (Listing S3) module is used. Assimulo was

437 used as an interface with ODE/DAE solvers such as SUNDIALS IDA (Hindmarsh et al., 2005)

438 or DASSL (Petzold, 1982). The solveDAE method forms the residual method and initial

439 values for the time, states and state derivatives required by a DAEs solver. The residual

440 method takes as input time $t$, state $y$, state derivative $y^{\prime}$ and returns a residual vector (zero if a

441 solution is found). Argument lists for the residual are prepared by the residualArgs

442 method. It is also possible to create user-defined functions state_events and

443 handle_event for event tracking and handling in discontinuous problems for Assimulo. 
444 Some problems in pycodyn, which is formulated using difference or differential equations, can

445 be solved symbolically using the SymPy functions solve and dsolve. In particular, the

446 solve function, which symbolically solves equations and systems of equations, helps to form

447 the ceqs f method mentioned above. The dsolve function solves any supported kind of

448 ODEs. Therefore, DAEs needs to be transformed into ODEs by simplification.

449

450 Use cases

451 For testing purposes of pycodyn, we consider models of sucker rod strings. Let's take a look at 452 the steel sucker rod string, in which the length is $1510 \mathrm{~m}$. Such a string and its practical

453 dynamometer card are described in (Belov, 1960). The upper section of the string consists of 695 $454 \mathrm{~m}$ rods with a diameter of $22 \mathrm{~mm}$, and the lower section consists of $815 \mathrm{~m}$ rods with a diameter 455 of $19 \mathrm{~mm}$. This string will have a total mass of $3961 \mathrm{~kg}$, a total weight in the liquid of $34687 \mathrm{~N}$, a 456 spring constant of $44650 \mathrm{~N} / \mathrm{m}$, a damping constant of $2121 \mathrm{~N} \cdot \mathrm{s} / \mathrm{m}$. Liquid weight above the pump 457 with a diameter of $43 \mathrm{~mm}$ will be $18499 \mathrm{~N}$.

458

\section{Use case 1: Simulation of free vibrations of the sucker rod string}

460 First, simulate the free vibrations of the string using the Modelica language. Initially, the string is 461 stretched by moving the lower end of the string (mass $1 . \mathrm{s}$ ) by $1 \mathrm{~m}$. After the lower end is

462 released, free vibrations will begin. Use the model (Listing S1) with parameter values:

463 mass1.m=3961.0, spring1. $c=44650.0$, spring1. $d=2120.7$, and with initial conditions: mass1.s=-1, mass1.v=0. Simulation options: stopTime $=10.0$, numberofIntervals $=500$, tolerance $=1 e-006$, method $=$ 'dassl'. Now perform the simulation of free vibrations of the sucker rod string in pycodyn (Fig. 1). In the separate module (Listing S4) create the components: spring-damper s 1 and mass $\mathrm{m} 1$. In round brackets, there are the values of the attributes - the name and the known parameters.

469

470

from pycodyn import *

$471 \mathrm{~s} 1=$ SpringDamper (name='s1', $c=44650.0, \mathrm{~d}=2120.7$ )

$472 m 1=\operatorname{Mass}\left(\right.$ name $={ }^{\prime} m 1 ', m=3961.0$ )

473

474 Create the list of additional equations, formed by connecting the flanges of the components.

475 Then create the object of the component system.

476

477

peqs $=$ s $1 \cdot$ pinEqs $(1,[\mathrm{~m} 1 \cdot$ pins $[0]])$

$478 s=$ System $(e l s=[s 1, m 1]$, eqs=peqs $)$

479

480 A list of the model equations can be printed using the command print ( . eqs). To obtain 481 equations only in the symbolic form, the numerical values of the constructor parameters $\mathrm{c}, \mathrm{d}, \mathrm{m}$ 482 should be replaced by None: 
484

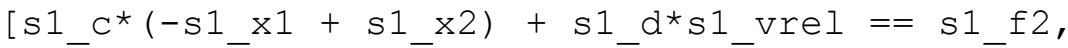

485

-s1_f2 = = s1_f1,

486

$\mathrm{s} 1 \_\mathrm{vrel}==-\left(\mathrm{s} 1 \_\mathrm{x} 1-\mathrm{s} 1 \_\mathrm{x} 1 \mathrm{p}\right) / \mathrm{dt}+\left(\mathrm{s} 1 \_\mathrm{x} 2-\mathrm{s} 1 \_\mathrm{x} 2 \mathrm{p}\right) / \mathrm{dt}$,

487 m1_a*m1_m ==m1_f1 $+\mathrm{ml}_{-} \mathrm{f2}$,

$488 \mathrm{~m} 1{ }_{-} \mathrm{a}==\left(\mathrm{m} 1_{-} \mathrm{v}-\mathrm{m} 1_{-} \mathrm{vp}\right) / \overline{\mathrm{d} t}$,

$489 \mathrm{~m} 1{ }_{-} \mathrm{v}==\left(\mathrm{m} 1_{-} \mathrm{x}-\mathrm{m} 1_{-} \mathrm{xp}\right) / \mathrm{dt}$,

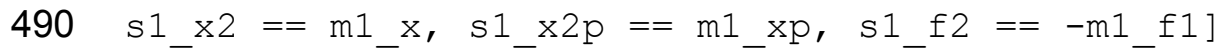

491

492

493

494

Solve the static problem — the string is stretched by $1 \mathrm{~m}$.

495

ics $=\{\mathrm{m} 1 . \mathrm{x}:-1.0, \mathrm{~m} 1 . \mathrm{v}: 0.0, \mathrm{~m} 1 . \mathrm{a}: 0.0, \mathrm{~s} 1 . \mathrm{x} 1: 0.0, \mathrm{~s} 1 . \mathrm{x} 1 \mathrm{p}: 0.0, \mathrm{~m} 1 . \mathrm{vp}: 0.0\}$

$d=s$. solve (ics)

496

497

The boundary conditions depend on the type of problem. If this is the problem of free

498

oscillations, then the position of the string top point elsd [ $\left.s 1^{\prime}\right] . x 1$ and the force on the

499

plunger elsd ['m1'].f2 are zero. Create the function to update the boundary conditions at

500

time $t$ for the $\mathrm{fnBC}$. vrs components. Then solve the dynamic problem - free vibrations of

501

the string.

502

503

def $\operatorname{fnBC}(d, t)$ :

504

$\mathrm{val}=0.0,0.0$

505

return dict(zip (fnBC.vrs, val))

506

fnBC.vrs $=\mathrm{s} . e l s d\left[\mathrm{sl}^{\prime}\right] . \mathrm{x} 1, \mathrm{~s} . \mathrm{elsd}\left[\mathrm{Iml}^{\prime}\right] . \mathrm{f} 2$

$507 \mathrm{~T}, \mathrm{R}=\mathrm{s}$. solveDyn (d, timeEnd=10, $\mathrm{fnBC}=\mathrm{fnBC}$ )

508

509

It is possible to improve the results in the Python model by using the more accurate but more

510 complex difference schemes. For example, if the trapezoidal rule is used (Listing S5), the second

511 and third equations for the Mass should be

512

513

$\operatorname{Eq}((\operatorname{self} . a+\operatorname{self} . a p) / 2,(\operatorname{self} . v-\operatorname{self} . v p) / d t)$,

$514 \operatorname{Eq}((\operatorname{self} \cdot v+\operatorname{self} . v p) / 2,(\operatorname{self} . x-\operatorname{self} . x p) / d t)$

515

516 Consider using components with DAEs and the Assimulo interface from the pycodynDAE

517 module (Listing S3). Create components and a system in the same way and first solve the static

518 problem - the string is stretched by $1 \mathrm{~m}$ (Listing S6).

519

520

521

$\mathrm{bc}=\{\mathrm{s} 1 . \times 1: 0.0, \mathrm{~s} 1 . \mathrm{D} \times 1: 0.0\} \#$ constant boundary conditions

eq $=\mathrm{s}$. eqs . subs $(\mathrm{bc})$

522 ics $=\{\mathrm{m} 1 . \mathrm{x}:-1.0, \mathrm{~m} 1 . \mathrm{v}: 0.0, \mathrm{~m} 1 . \mathrm{a}: 0.0, \mathrm{~s} 1 . \mathrm{Dx} 2: 0.0\}$

523 state $=$ s.solve (eq, ics)

524

525

526

Use the SUNDIALS IDA solver with absolute and relative tolerances 1e-06 to solve the dynamic problem - free vibrations of the string. 
527

528

529

530

531

532

533

534

535

536

537

538

539

540

541

542

543

544

545

546

547

548

549

550

551

552

553

554

555

556

557

558

559

560

561

562

563

564

565

566

567

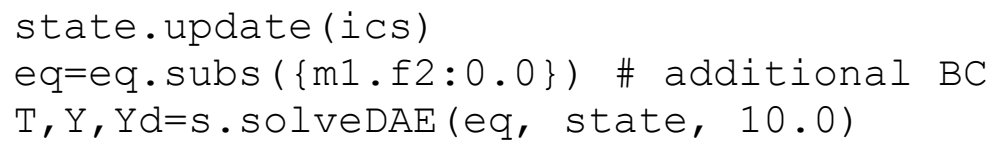

Now solve the equations symbolically using the ODE-solver SymPy (Listing S7). Substitute the boundary conditions into the system of equations (m1.f2 $2=0.0, \mathrm{~s} 1 . \mathrm{x} 1=0.0$, $\mathrm{s} 1 . \mathrm{Dx} 1=0.0)$, simplify the system, and after substitution of functions and derivatives instead of symbols, obtain the well-known equations of the harmonic oscillator.

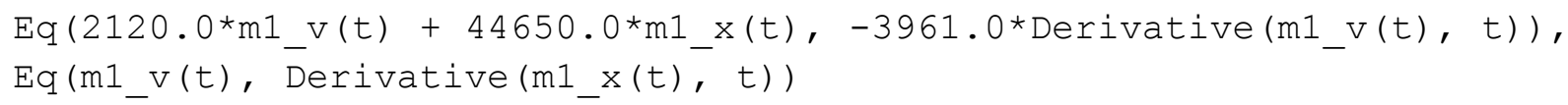

Solve the equation using dsolve with the initial conditions $\mathrm{m}{ }_{-} \mathrm{x}(0)=-1.0, \mathrm{~m}{ }_{-} \mathrm{v}(0)$ $=0.0$ and obtain a well-known solution:

$E q\left(m 1 \_v(t), 3.36816 * \exp (-0.26761 * t) * \sin (3.34676 * t)\right)$, Eq $\left(m 1 \_x(t),-(0.08 * \sin (3.34676 * t)+\cos (3.34676 * t)) * \exp (-0.26761 * t)\right.$

\section{Use case 2: Simulation of the pumping process}

During pumping, the following loads act on the string: the weight of the rods, the weight of the liquid (only during the upstroke) and dynamic loads (Belov, 1960; Gibbs, 2012). To build the single-section model Pumping in Modelica (Listing S1), use the components of the oscillator and additional components: motion 1 (describes the movement of the upper point) and forcel (describes the forces acting on the lower point). This is a simplified model that does not take into account other types of loads (Kopey VB, Kopey BV \& Kuzmin, 2017). The stroke length of the upper point is $2.1 \mathrm{~m}$, the number of double strokes per minute is 6.4. During the downstroke, the weight of the rods acts on the lower point. During the upstroke, the weight of the liquid is added to it. This is shown in the algorithm section of the Pumping model:

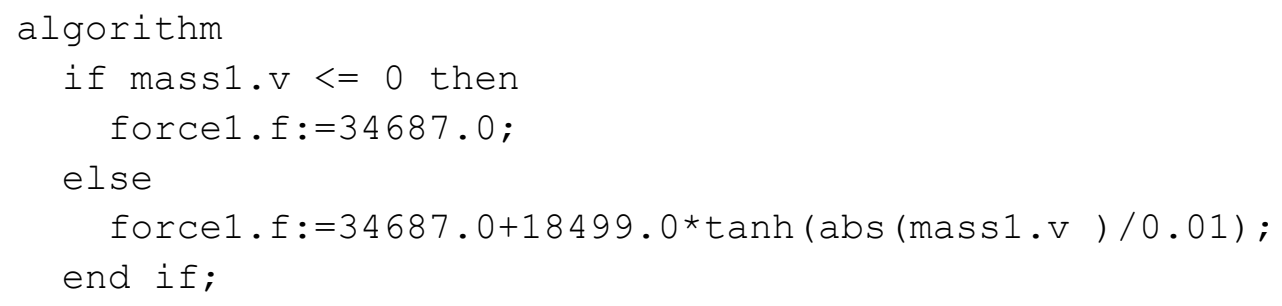

The analog of this single-section model in Python is shown in (Listing S8). Now in the new module (Listing S9) create the two-section Python-model of the sucker rod string, in which each section of the string is modeled by three components. The model of each section consists of three 1D mechanical translational components: SpringDamper, Mass and Force (Fig. 2). The 
568 SpringDamper component is designed to simulate the elastic-damper properties of the string 569 section, the Mass component simulates the inertial properties of the section, and the Force 570 component simulates the section weight in the fluid and other external forces acting on the 571 section. The upper section has a mass of $2112 \mathrm{~kg}$, a weight in the liquid of $18494 \mathrm{~N}$, a spring 572 constant of $114926 \mathrm{~N} / \mathrm{m}$, a damping constant of $5458 \mathrm{~N} \cdot \mathrm{s} / \mathrm{m}$. The lower section has a mass of

$5731850 \mathrm{~kg}$, a weight in the liquid of $16193 \mathrm{~N}$, a spring constant of $73021 \mathrm{~N} / \mathrm{m}$, a damping constant 574 of $3468 \mathrm{~N} \cdot \mathrm{s} / \mathrm{m}$. Assign values to the variable of sections weights $\mathrm{fs}$ and the variable of liquid 575 weight above the plunger $\mathrm{f} r$.

576

577 from pycodyn import *

$578 f_{S}=(-18494.0,-16193.0)$

579 fr $=-18499.0$

580

581 Create the components: the spring-damper of the first (upper) section $\mathrm{s} 1$, the mass of the first 582 section $\mathrm{m} 1$, the weight of the first section $\mathrm{f} 1$, the spring-damper of the second section $\mathrm{s} 2$, the

583

584

585

586

587

588

589

590

591

592

593

594

595

596

597

598

599

600

601

602

603

604

605

606

607

608

609

610 mass of the second section $\mathrm{m} 2$, the weight of the second section with the weight of the liquid $\mathrm{f} 2$.

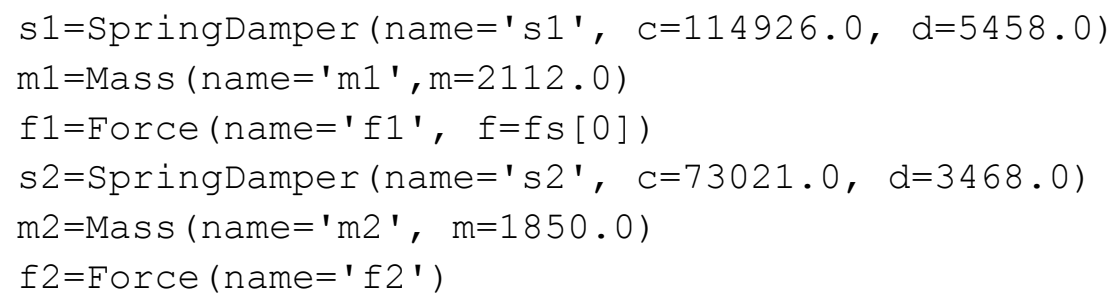

Create the list of the additional equations (formed by connecting the component flanges) and the object of the component system (the string model).

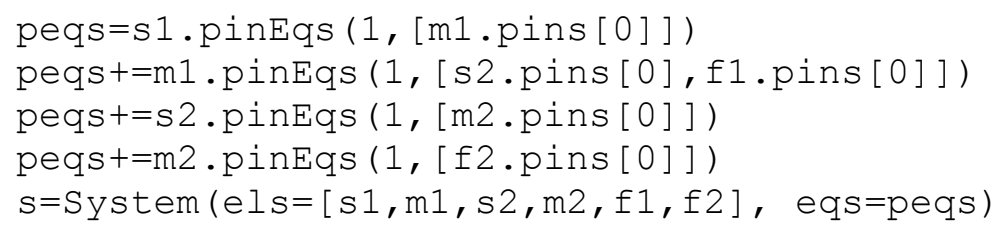

The complete list of equations for this system s.eqs in the SymPy format:

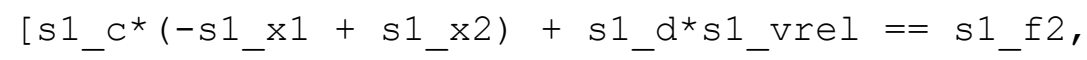

$-s 1_{-} f 2=s 1_{-} f 1$,

$\mathrm{s} 1$ vrel $==-\left(\mathrm{s} 1 \mathrm{x}_{1}-\mathrm{s}{ }_{-} \mathrm{x} 1 \mathrm{p}\right) / \mathrm{dt}+\left(\mathrm{s} 1_{-} \mathrm{x} 2-\mathrm{s}{ }_{-} \mathrm{x} 2 \mathrm{p}\right) / \mathrm{dt}$,

$m 1 \_a * m 1 \_m=m 1_{-} f 1+m 1_{-} f 2$,

$\mathrm{m} 1 \_\mathrm{a}==\left(\mathrm{m} 1_{-} \mathrm{v}-\mathrm{m}{ }_{-} \mathrm{vp}\right) / \mathrm{dt}$,

$\mathrm{m} 1 \_\mathrm{v}==\left(\mathrm{m} 1_{-} \mathrm{x}-\mathrm{m} 1_{-} \mathrm{xp}\right) / \mathrm{dt}$,

$s 2 c^{*}\left(-s 2 \times x 1+s 2 \_x 2\right)+s 2 d^{*} s 2$ vrel == s2 f 2 ,

$-s 2 \_f 2==s 2 f 1$, 


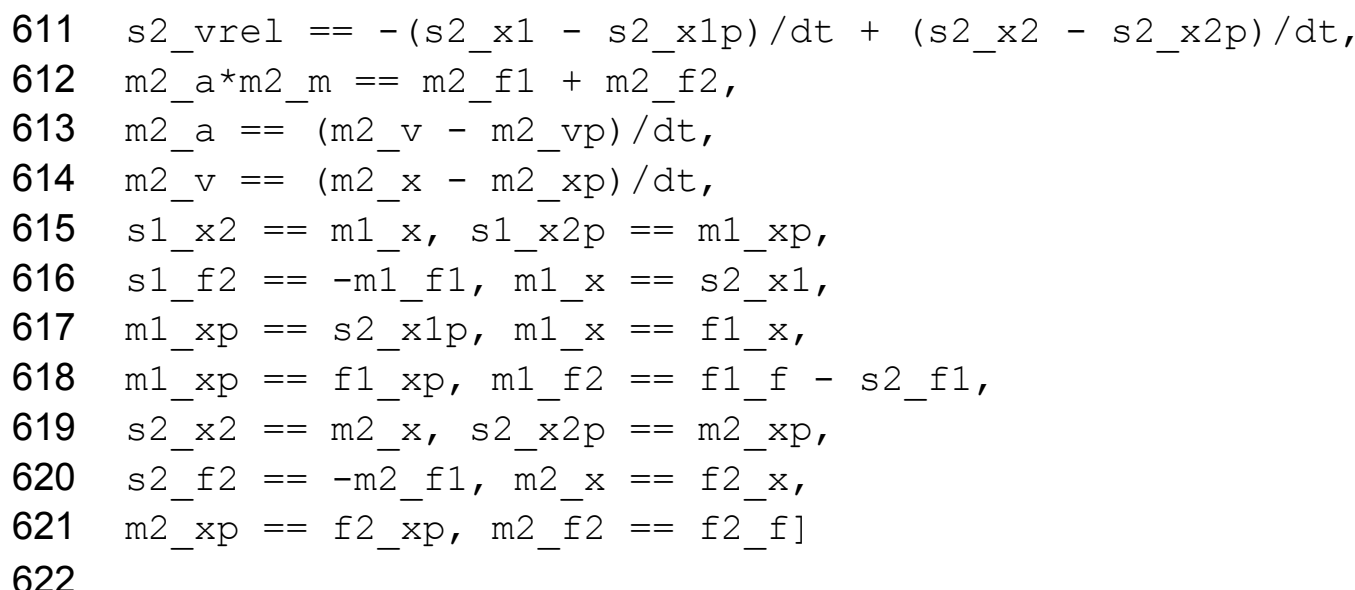

623 Solve the static problem — the string under the maximum static loads.

624

625

626

ics $=\{\mathrm{m} 1 . \mathrm{v}: 0.0, \mathrm{~m} 1 . \mathrm{a}: 0.0, \mathrm{~m} 2 . \mathrm{v}: 0.0, \mathrm{~m} 2 . \mathrm{a}: 0.0, \mathrm{~s} 1 . \mathrm{x} 1: 0.0, \mathrm{~s} 1 . \mathrm{x} 1 \mathrm{p}: 0.0$,

$627 \mathrm{~d}=\mathrm{s} . \mathrm{sol}$ ve (ics)

628

629 Dictionary d contains the results. To display the position value for the bottom point of the 630 second section, enter the command print $(d[m 2 . x])$. We get the result -0.94 . This is the 631 elongation value of the string under the maximum load.

632 Solve the dynamic problem - the upper point has a harmonic motion. The motion function 633 describes the harmonic motion of the upper point and returns its position at time $t$.

634

635

636

639

640 The force function returns the value of the force on the pump plunger $\mathrm{F}$, depending on the

641 value of its speed $v$. If the speed is less than zero (downstroke of the string), the function returns

642 the weight value of the second section. Otherwise, the function returns the sum of the second

643 section weight and the liquid weight above the plunger. This function should be smoothed when

644 the sign of the velocity changes, for example, using the math. tanh hyperbolic tangent

645 function.

646

647 def force(v):

648 F=fs[1] \# weight of the second section

649 if $\mathrm{v}>0$ : \# if upstroke

650

651

$\mathrm{F}+=\mathrm{fr} \#$ increase the force by value of the fluid weight

652 return $F^{*}$ math.tanh (abs $\left.(v) / 0.01\right)$ \# smoothing near the point $v=0$ 
653 Create the function to update the boundary conditions at time $t$ for $f n B C . v r s$ components.

654 Here $d$ is the dictionary of the results calculated in the previous step. Then solve the problem.

655

656

657

658

def $f n B C(d, t):$

659

$\operatorname{val}=\operatorname{motion}(t)$, force $(d[m 2 . v])$

return dict (zip(fnBC.vrs, val))

fnBC.vrs $=$ s.elsd['s1'].x1, s.elsd['f2'].f

660

$T, R=s . s o l v e D y n(d$, timeEnd $=20.0$, $f n B C=f n B C)$

661

662

The simulation of the variable structure system (breakage of the second section) by the Euler

663 method ( $d t=0.1)$ is implemented in (Listing S10). If the force at the top of the second section is

664 greater than $56000 \mathrm{~N}$, then the section breaks off. The user method event is created to handle

665 the event. At each step, this method checks the condition state [s1.f1]>56000. If the result is True, then an event occurs. The handling of this event consists in changing the components of the system (only $s 1, m 1, f 1$ remain after the breakage), changing additional equations at the connection points, changing the boundary conditions $\mathrm{fnBC}$ (the weight of the second section and

669

670

671

672

673

674

675

676

677

678

679

680

681

682

683

684

685

686

687

688

689

690

691

692

693

the weight of the liquid are zero). The ceqs $f$ method is also updated by calling

createCurEqs.

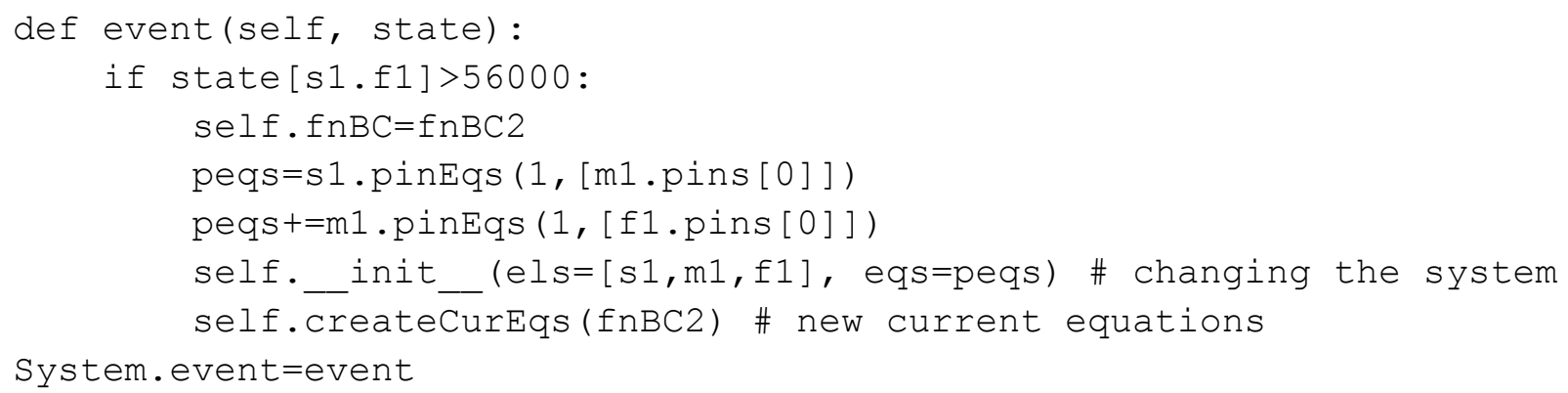

Analogous single-section (Listing S11) and two-section (Listing S12) models, based on differential equations, are developed using the pycodynDAE module. The DAE system should be supplemented with equations that describe the position of the upper point and the force that acts on the lower point. For example:

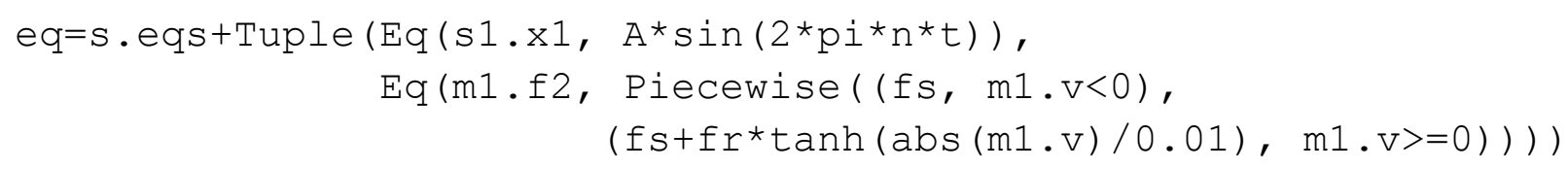

Alternatively, you can replace the symbols $\mathrm{s} 1 . \mathrm{x} 1$ and $\mathrm{m} 1 . \mathrm{f} 2$ with the right-hand sides of these equations.

\section{Simulation results}


694 The results of the simulation of free vibrations are shown in Fig. 3. The frequency of free

695

696

697

698

699

700

701

702

703

704

705

706

707

708

709

710

711

712

713

714

715

716

\section{7}

718

719

720

721

722

723

724

725

726

727

728

729

730

731

732

733

vibrations corresponds to the theoretical natural frequency of the harmonic oscillator $\omega=(j / m)^{1 / 2}=(44650 / 3961)^{1 / 2}=3.357 \mathrm{rad} / \mathrm{s}$, where $j$ is the stiffness, $m$ is the mass. Such vibrations occur during normal operation of the pump due to the sharp removal or application of the load (the pump valve opens or closes) and are noticeable in the upper and lower parts of the dynamometer card (Belov, 1960). The differences are explained by the use of unequal difference schemes. The results obtained analytically and by IDA/DASSL solvers are almost equal, therefore they are shown by a single curve in the figure. The global error values (at $t=1 \mathrm{~s})$ for the Euler $(d t=0.1)$, trapezoidal rule $(d t=0.1)$, Euler $(d t=0.01)$, DASSL (OpenModelica), SUNDIALS IDA methods are respectively $0.344,0.034,0.046,6.35 \mathrm{E}-05,1.46 \mathrm{E}-05$. The simulation time is $0.25,0.5,2.46,0.29,0.028$ seconds, respectively. The total time of the module execution (total simulation time for OpenModelica) is 4.1, 5.5, 6.3, 5.1, 1.9 seconds, respectively. These data were obtained for this configuration: simulation interval 0-10 seconds, CPU 2.5 GHz, Python 3.7, NumPy 1.16.4, OpenModelica 1.12, Sundials 2.6.

The results of the pumping process simulation (Fig. 4) correspond to practical dynamometer cards obtained on real wells (Belov, 1960). Single-section (Listing S8) and two-section (Listing S9) models, simulated by the Euler method $(\mathrm{dt}=0.1)$, somewhat distort the right and left sides of the card and smooth the upper and lower sides (Fig. 4A). Single-section (Listing S11) and twosection (Listing S12) models, simulated by the IDA, give somewhat larger values of maximum load and lower values of minimum load (Fig. 4B). The two-section model is more adequate. The dynamometer card of the string breakage model (Fig. 5) corresponds to practical dynamometer cards with their typical flat shapes (Belov, 1960).

\section{Discussion}

It is noticeable that the global error for the Euler method ( $d t=0.1)$ is much larger, therefore it is not advisable to use it for the free vibration problem. In addition, numerical solution procedure in pycodyn has a low performance if difference equations are used. In the future, it is planned to improve performance, for example by using Cython.

The differences with the practical dynamometer card are explained by the fact that the real string has a greater number of degrees of freedom. For a more adequate simulation, you need to increase the number of sections of the model (Kopey VB, Kopey BV \& Kuzmin, 2017) or use the wave equation, which is a partial differential equation (Gibbs, 2012). In addition, it is difficult to know the exact value of the damping constant, which depends on many factors (Kopey VB, Kopey BV \& Kuzmin, 2017). In general, all these models can be used for approximate modeling of the pumping process.

The Python language allows a simple modification and improvement of pycodyn. In the future, it is planned to extend the set of the components (e.g. create electrical and hydraulic components), develop support for hierarchical subsystems and the tools for building models using component diagrams. To implement hierarchical subsystems, you can move functions for solving equations to a separate module and add pins to the System class. The problem in the form of difference

Peer] Comput. Sci. reviewing PDF | (CS-2019:03:35946:2:0:NEW 14 Sep 2019) 
734 equations is usually more difficult to formulate. But SymPy can be used to automate the

735 conversion of differential equations to difference equations. In order to simplify the code of the

736 pycodyn and pycodynDAE modules, the algorithms for equations sorting, eliminating, and

737 simplifying are not implemented. This is planned to be implemented in the future using SymPy.

738

739

\section{Conclusions}

740 The Python-classes that allow creating the Modelica-like models in Python without the need to

741

742

743

744

745

746

747

748

749

750

751

752

753

754

755

756

757

758

759

760

761

762

763

764

765

766

767

768

769

770

771

772 study and apply specialized modeling languages are developed. The suggested approach simplifies the understanding of the system, its modification and improvement, adaptation for other purposes, makes it available to a much larger community, simplifies integration into thirdparty software.

Difference or differential equations can be used to describe components. Using difference equations and the pycodyn module allows simplifying the implementation of the hybrid modeling, variable structure systems modeling and the requirements for the modules for symbolic mathematics and for solving equations. It is also well suited for experimenting with evolutions of modeling capabilities. In particular, one can experiment with arbitrary difference schemes, make arbitrary symbolic manipulations and modify numerical solution procedures. However, the pycodynDAE module can provide higher accuracy and performance using thirdparty DAEs solvers that are suitable for stiff problems. With SymPy, some tasks can be solved symbolically.

The comparison of simulation results of sucker rod string with practical dynamometer cards and Modelica models verify the adequacy of the models. The pycodyn framework can be used to study the principles of component-oriented modeling and for various kinds of experiments on its new features. The source code is freely available under the GNU GPLv3 open-source license from the GitHub (https://github.com/vkopey/pycodyn).

\section{References}

Åkesson J, Årzén K-E, Gäfvert M, Bergdahl T, Tummescheit H. 2010. Modeling and Optimization with Optimica and JModelica.org — Languages and Tools for Solving Large-Scale Dynamic Optimization Problems. Computers and Chemical Engineering, 34(11): 1737-1749 DOI: 10.1016/j.compchemeng.2009.11.011

Andersson C, Führer C, Åkesson J. 2015. Assimulo: A unified framework for ODE solvers. Mathematics and Computers in Simulation 116:26-43 DOI: 10.1016/j.matcom.2015.04.007. Atkinson KA. 1989. An Introduction to Numerical Analysis (2nd ed.). New York: John Wiley \& Sons. ISBN 978-0-471-50023-0

Barton PI, Pantelides CC. 1993. gPROMS-a combined discrete/continuous modelling environment for chemical processing systems. Simulation Series 25:25-34

Beal LDR, Hill D, Martin RA, Hedengren JD. 2018. GEKKO Optimization Suite. Processes 6(8) DOI: $10.3390 / p r 6080106$. 
773 Belov IG. 1960. Study of the operation of downhole pumps by a dynamograph. Moscow:

774 GOSTOPTEHIZDAT.

775 Benvenuti L, Bresolin D, Collins P, Ferrari A, Geretti L, Villa T. 2014. Assume-guarantee

776 verification of nonlinear hybrid systems with Ariadne. Int. J. Robust Nonlinear Control 24:699-

777724 DOI: $10.1002 /$ rnc.2914

778 Broman D. 2010. Meta-Languages and Semantics for Equation-Based Modeling and

779 Simulation. PhD thesis, Thesis No 1333. Department of Computer and Information Science,

780 Linköping University, Sweden.

781 Clewley RH, Sherwood WE, LaMar MD, Guckenheimer JM. 2007. PyDSTool, a software

782 environment for dynamical systems modeling. Available at http://pydstool.sourceforge.net

783 (accessed 16 March 2019).

784 Elmqvist H, Henningsson T, Otter M. 2016. Systems Modeling and Programming in a Unified

785 Environment Based on Julia. In: Margaria T., Steffen B. (eds) Leveraging Applications of

786 Formal Methods, Verification and Validation: Discussion, Dissemination, Applications. ISoLA

787 2016. Lecture Notes in Computer Science 9953. Cham: Springer DOI: 10.1007/978-3-319-

788 47169-3 15

789 Elmqvist H. 1978. A Structured Model Language for Large Continuous Systems. Department of

790 Automatic Control, Lund Institute of Technology (LTH).

791 Fritzson P, Aronsson P, Lundvall H, Nyström K, Pop A, Saldamli L, Broman D. 2005. The

792 OpenModelica modeling, simulation, and development environment. In: 46th Conference on

793 Simulation and Modelling of the Scandinavian Simulation Society (SIMS2005), Trondheim,

794 Norway, October 13-14, 2005.

795 Fritzson P, Broman D, Cellier F. 2009. Equation-Based Object-Oriented Languages and Tools.

796 In: Eugster P, ed. Object-Oriented Technology. ECOOP 2008 Workshop Reader. ECOOP 2008.

797 Lecture Notes in Computer Science 5475. Berlin, Heidelberg: Springer, 18-29 DOI:

798 10.1007/978-3-642-02047-6_3.

799 Fritzson P, Engelson V. 1998. Modelica - a unified object-oriented language for system

800 modeling and simulation. In: Jul E, ed. ECOOP'98-Object-Oriented Programming. Lecture

801 Notes in Computer Science 1445:67-90, Berlin Heidelberg: Springer.

802 Fritzson PA. 2015. Principles of Object Oriented Modeling and Simulation with Modelica 3.3:

803 A Cyber-Physical Approach. 2nd edition. Wiley-IEEE Press.

804 Gibbs SG. 2012. Rod Pumping: Modern Methods of Design, Diagnosis and Surveillance.

805 Publisher: Author. ISBN-13: 978-0-984-9661-0-3.

806 Hedengren JD, Shishavan RA, Powell KM, Edgar TF. 2014. Nonlinear modeling, estimation 807 and predictive control in APMonitor. Computers and Chemical Engineering. 70:133-148 DOI:

808 10.1016/j.compchemeng.2014.04.013.

809 Hindmarsh AC, Brown PN, Grant KE, Lee SL, Serban R, Shumaker DE, Woodward CS.

810 2005. SUNDIALS: Suite of Nonlinear and Differential/Algebraic Equation Solvers $A C M$

811 Transactions on Mathematical Software 31(3):363-396 Also available as LLNL technical report

812 UCRL-JP-200037. 
813 Kopey VB, Kopey BV, Kuzmin OO. 2017. Design principles of the Model of Sucker-Rod 814 Pumping System for Maplesoft MapleSim 7 environment Scientific bulletin Ivano-Frankivsk

815 National Technical University of Oil and Gas 2:42-52

816 http://elar.nung.edu.ua/handle/123456789/6568

817 Margolis B. 2017. SimuPy: A Python framework for modeling and simulating dynamical 818 systems. The Journal of Open Source Software 2(17):396 DOI: 10.21105/joss.00396.

819 Meurer A, Smith CP, Paprocki M, Čertík O, Kirpichev SB, Rocklin M, Kumar A, Ivanov 820 S, Moore JK, Singh S, Rathnayake T, Vig S, Granger BE, Muller RP, Bonazzi F, Gupta H, 821 Vats S, Johansson F, Pedregosa F, Curry MJ, Terrel AR, Roučka Š, Saboo A, Fernando I, 822 Kulal S, Cimrman R, Scopatz A. 2017. SymPy: symbolic computing in Python. PeerJ 823 Computer Science 3:e103 DOI: 10.7717/peerj-cs.103.

824 Nikolić DD. 2016. DAE Tools: equation-based object-oriented modelling, simulation and 825 optimisation software. PeerJ Computer Science 2:e54 DOI: 10.7717/peerj-cs.54

826 Oliphant TE. 2007. Python for Scientific Computing Computing in Science \& Engineering 9:

827 10-20 DOI:10.1109/MCSE.2007.58

828 Petzold L. 1982. Description of DASSL: A differential/algebraic system solver. SAND82-8637.

829 Piela P, McKelvey R, Westerberg A. 1993. An Introduction to the ASCEND Modeling

830 System: Its Language and Interactive Environment. Journal of Management Information

831 Systems. 9:91-122 DOI: 10.1080/07421222.1992.11517969.

832 Pop A, Fritzson P, Remar A, Jagudin E, Akhvlediani D. 2006. OpenModelica Development 833 Environment with Eclipse Integration for Browsing, Modeling, and Debugging. In: Kral C and 834 Haumer A, ed. Proceedings of the 5th International Modelica Conference, Vienna, Austria, 835 September 2006.

836 Runge C. 1895. Math. Ann. 46:167 DOI: 10.1007/BF01446807.

837 Short T. 2017. Equation-based modeling and simulations in Julia. Available at 838 https://github.com/tshort/Sims.jl (accessed 16 March 2019).

839 Van Rossum G, Drake JrFL. 1995. Python reference manual. Amsterdam: Centrum voor 840 Wiskunde en Informatica. 


\section{Table $\mathbf{1}$ (on next page)}

The Mass class in Python (on the left) in comparison with the same class in Modelica (on the right). 


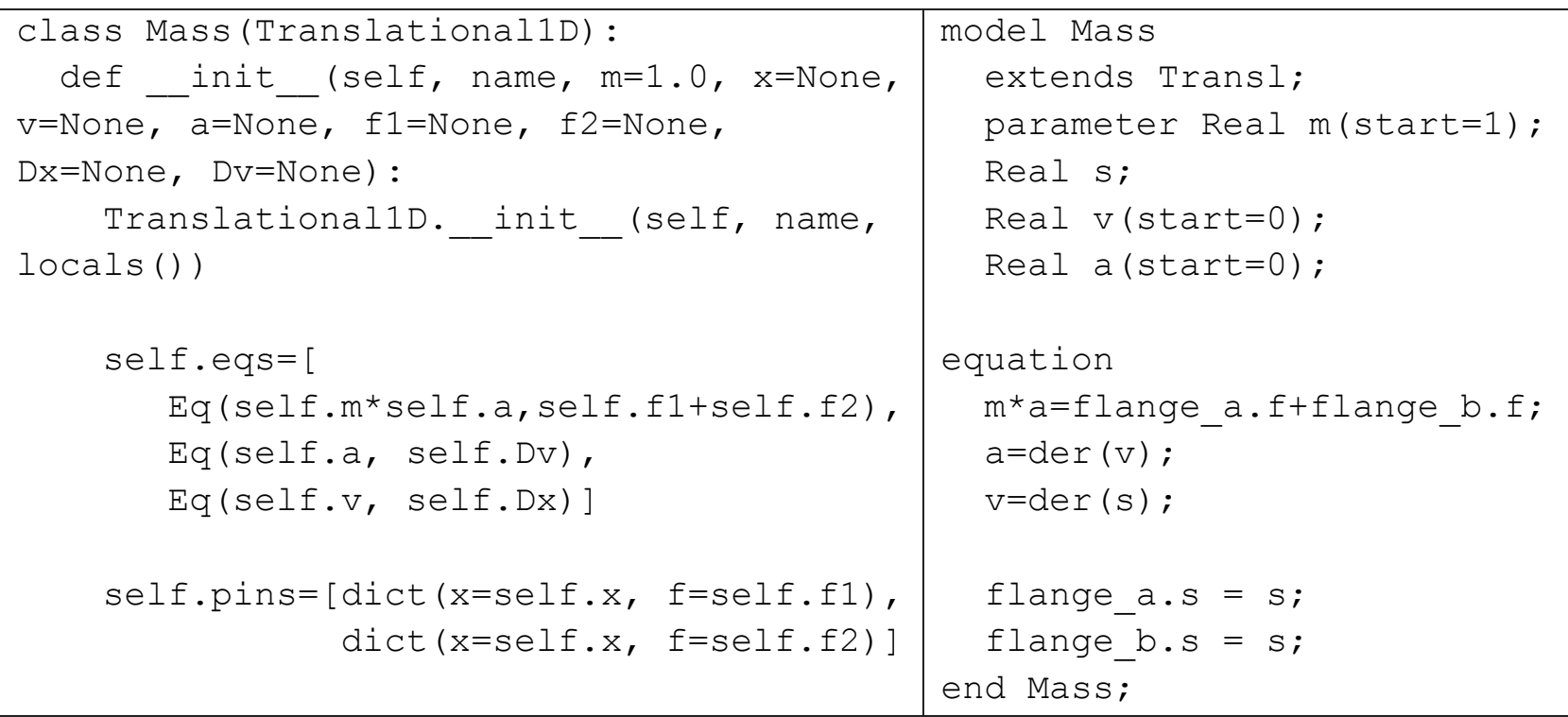


Figure 1

Component diagram of the oscillator model.

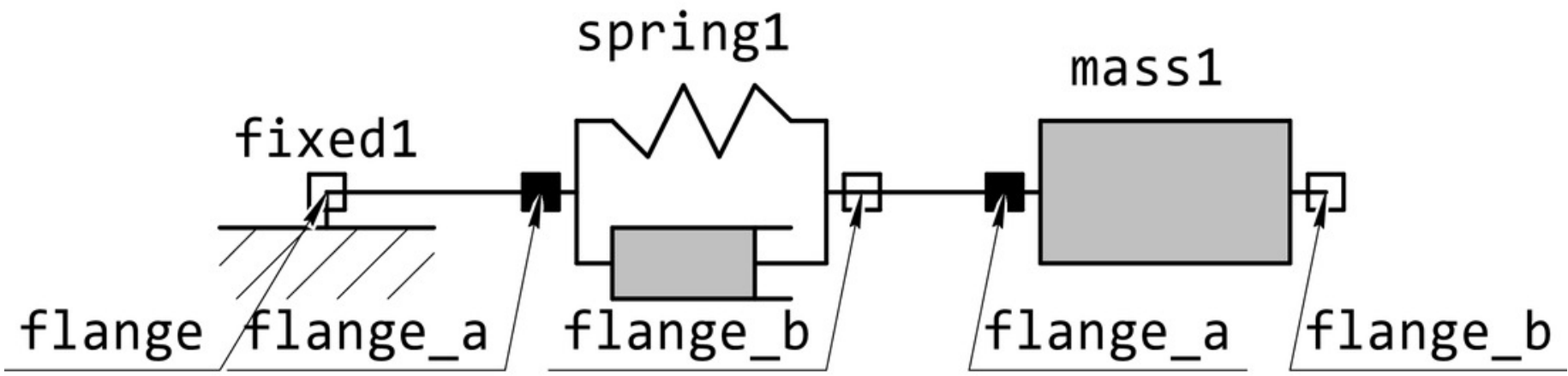


Figure 2

Component diagram of the model with two sections.

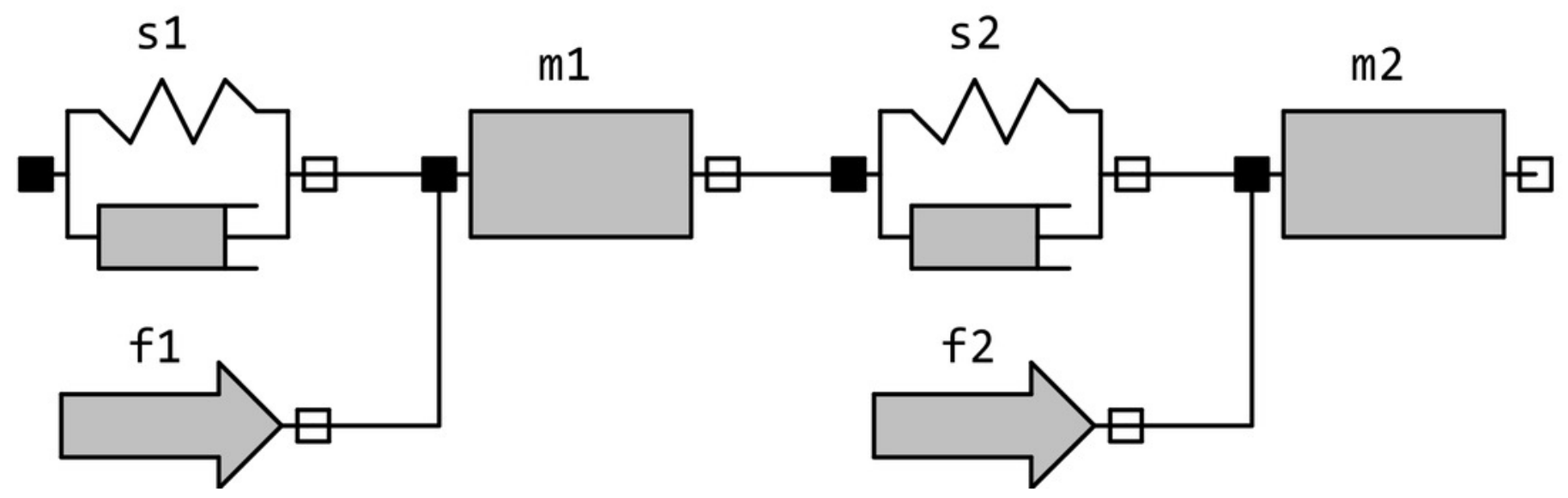


Figure 3

Plunger position $(x)$ during free oscillation of the string.

(A) Euler method with time step $\mathrm{dt}=0.1 \mathrm{~s}$; (B) Euler method with time step $\mathrm{dt}=0.01 \mathrm{~s}$; (C) Trapezoidal rule with time step $d t=0.1$ s; (D) DASSL (Modelica-model), SUNDIALS IDA, analytical solution.

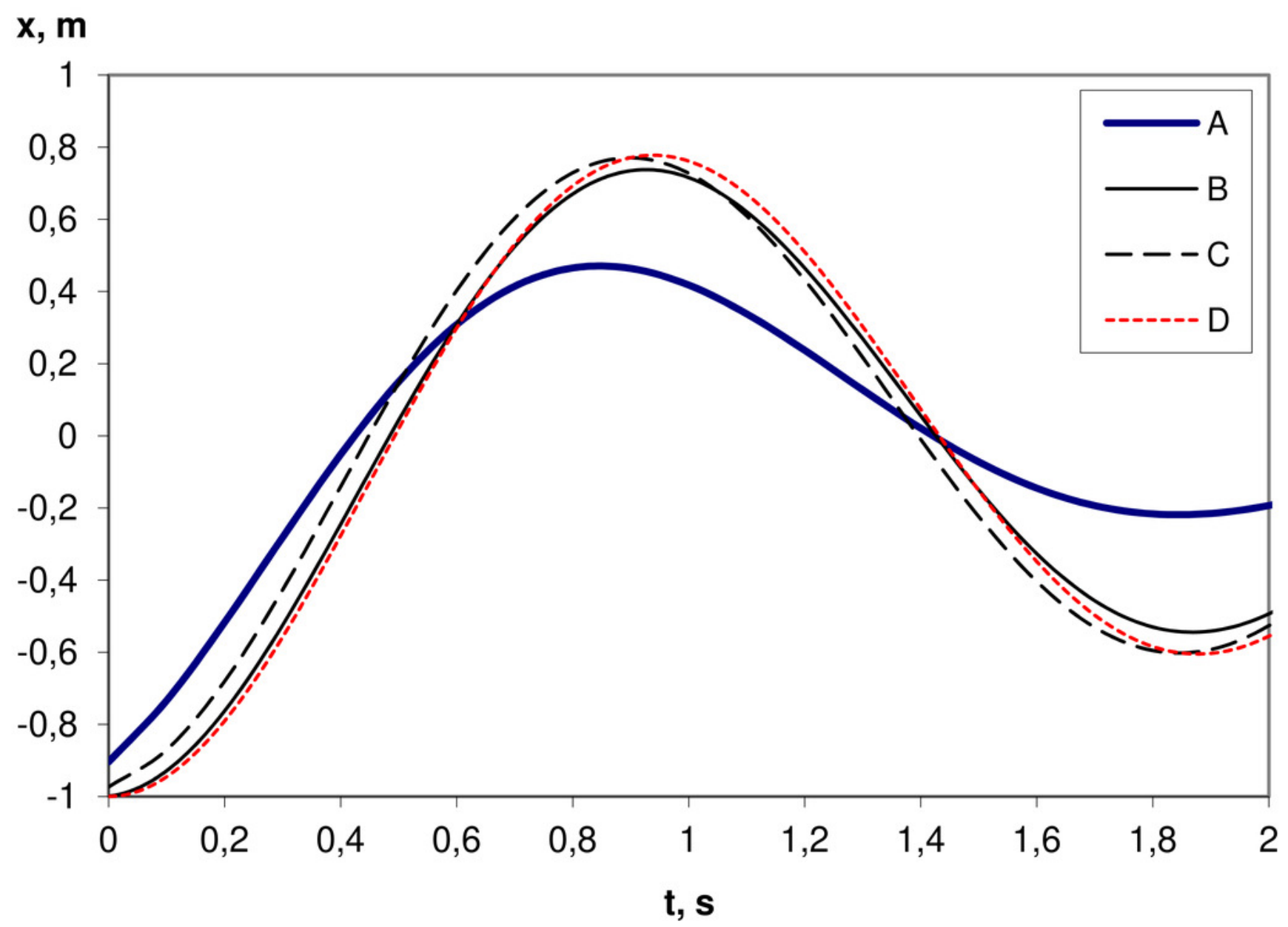




\section{Figure 4}

Simulation results - wellhead dynamometer cards.

(1) practical dynamometer card; (2) two-section model; (3) single-section model; (A) pycodyn with Euler method, $d t=0.1$; (B) pycodynDAE with SUNDIALS IDA. 

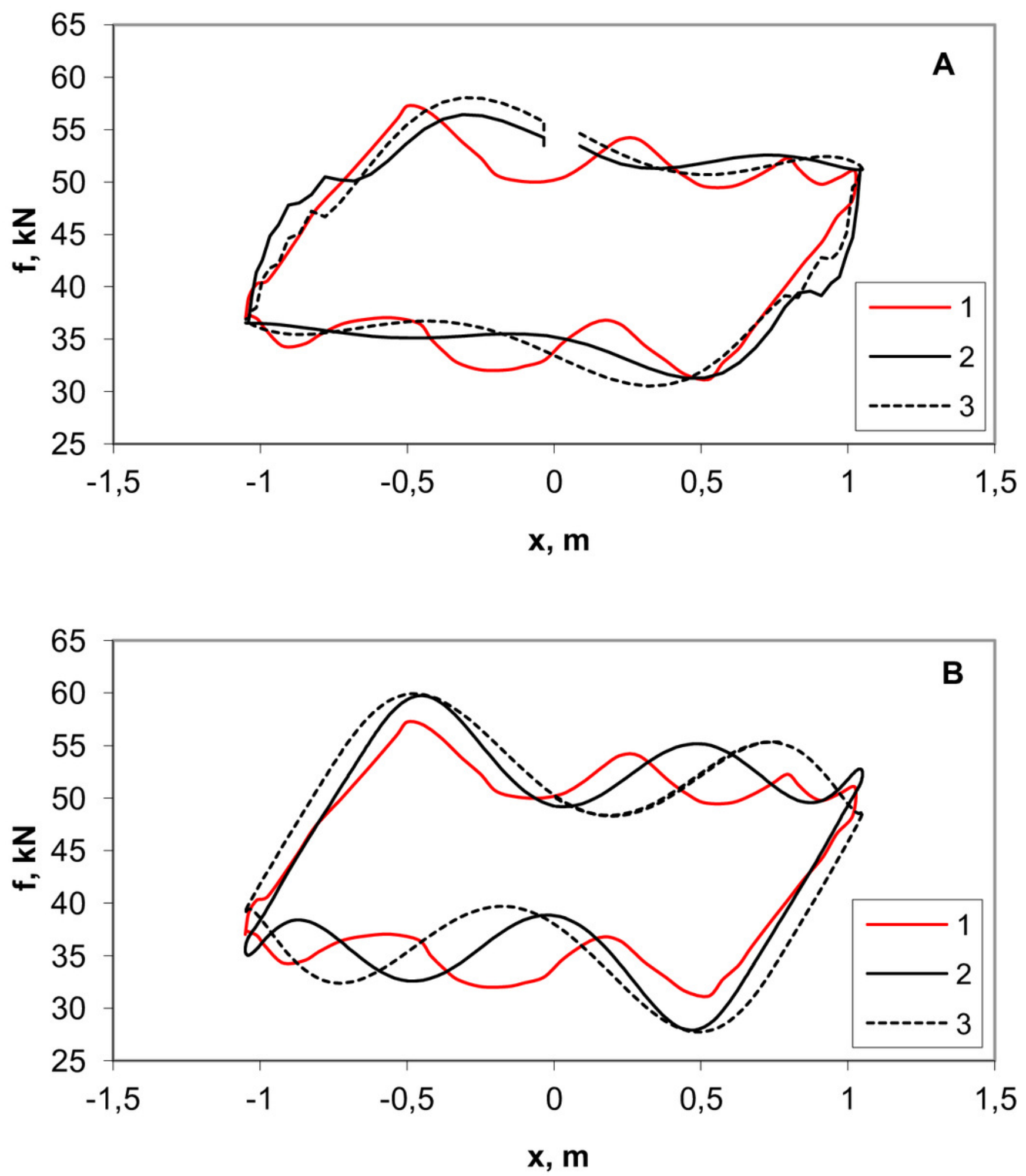
Figure 5

The simulation of the breakage of the sucker rod string (wellhead dynamometer card).

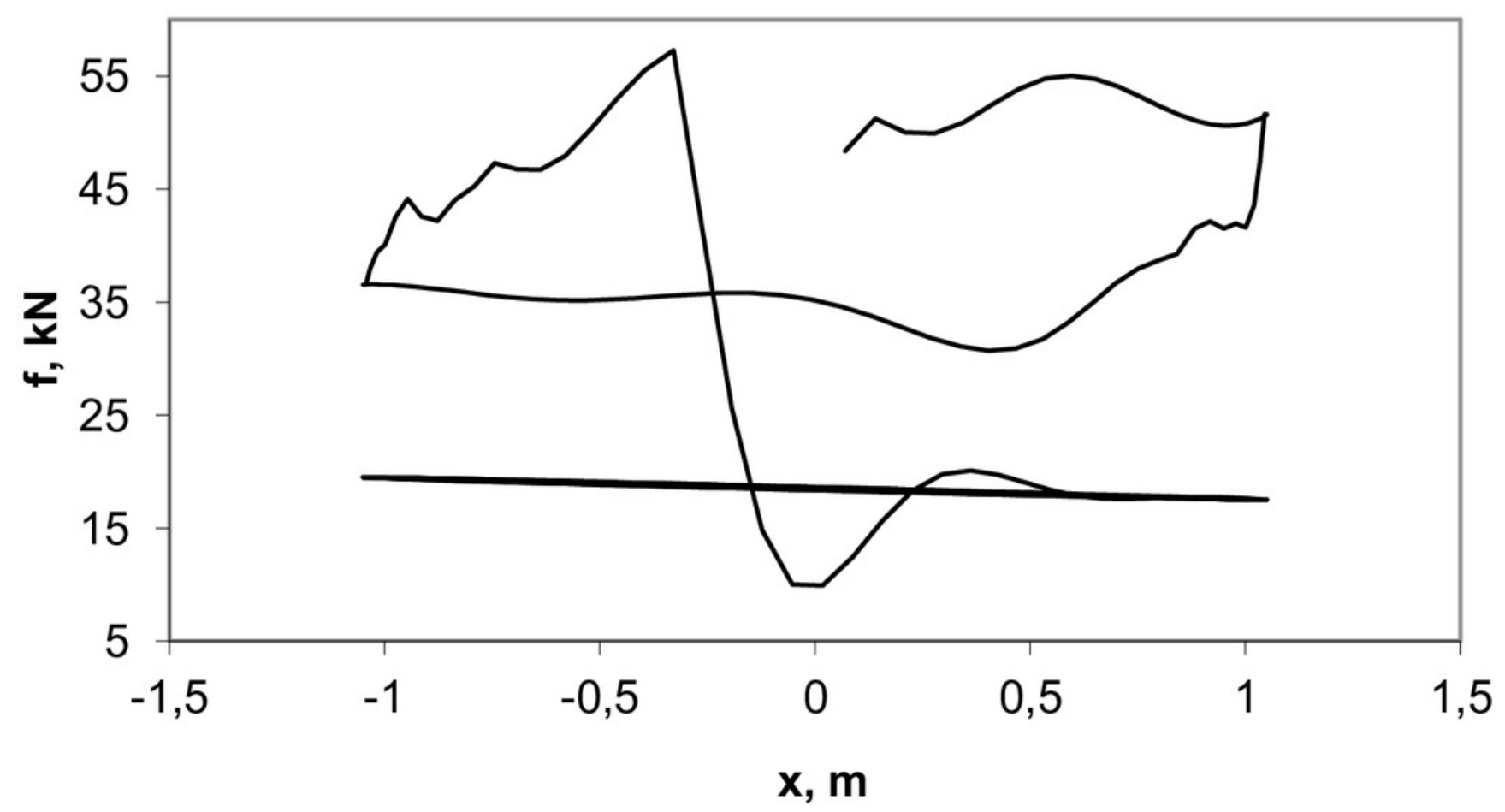

